\title{
Exploring the Intersection Between Diet and Self- Identity: A Cross-Sectional Study With Australian Adults
}

\author{
Jillian Claire Ryan, BPysch(Hons), $\mathrm{PhD}^{1}$; Caitlyn Alchin ${ }^{2}$; Kim Anastasiou, BND(Hons) ${ }^{3}$; \\ Gilly Hendrie, BSc(Dietetics), BSc(Hons), $\mathrm{PhD}^{3}$; Sarah Mellish, BPsych(Hons), $\mathrm{PhD}^{2}$; \\ Carla Litchfield, BSc(Hons), $\mathrm{PhD}^{2}$
}

\begin{abstract}
Objective: Diet-related self-identity, which includes components such as individuals' overall dietary pattern and food choice motivations, is a strong predictor of health behaviors. This study sought to assess the variation in dietary patterns reported by a sample of Australian adults and their associations with diet quality.

Design: Cross-sectional survey.

Participants: Australian adults $(\mathrm{n}=2,010)$

Variables measured: The main outcome measure was diet quality relative to the Australian Dietary Guidelines, measured by the Healthy Diet Score survey. Other outcomes captured included dietary patterns (eg, unrestricted, vegetarian, flexitarian, or ketogenic diets), diet-related self-identity constructs (centrality, prosocial motivation, personal motivation, and strictness), and sociodemographic characteristics (eg, age, sex, and education level).

Analysis: Data were analyzed descriptively, and ordinary least squares regression was performed to identify significant predictors of diet quality.

Results: Eighteen unique dietary patterns were reported. These were classified into 3 categories on the basis of the degree of restriction of core food groups. Diets based on restriction of animal protein were associated with the highest diet quality, including the highest consumption of fruits, vegetables, and whole grains, whereas restriction of other foods was associated with the poorest diet quality. Unrestricted diets reported the highest consumption of discretionary food (high in saturated fat, salt, or added sugar). Finally, the regression analysis found that diet quality was significantly predicted by dietary pattern and diet-related self-identity constructs $\left(F[8,1974]=54.952 ; P<0.0001\right.$; adjusted $\left.R^{2}=0.179\right)$.

Conclusions and Implications: Dietary pattern and diet-related self-identity constructs are key determinants of diet quality. This has implications for future interventions, including that programs and messages could be tailored to ensure they align with the target population's self-identity and overall dietary patterns. Key Words: diet self-identity, public health, psychological determinants of diet, food choice (J Nutr Educ Behav. 2022;54:20-27.)
\end{abstract}

Accepted August 3, 2021. Published online September 20, 2021.

\section{INTRODUCTION}

The past half-century has seen a major shift in food systems. Globally, consumption of processed and ultraprocessed foods has increased, whereas consumption of unprocessed and minimally processed

\footnotetext{
${ }^{1}$ Precision Health Future Science Platform, Commonwealth Scientific and Industrial Research Organisation, Adelaide, Australia

${ }^{2}$ Conservation Psychology and Applied Animal Behaviour Research Group, University of South Australia, Magill Campus, Adelaide, Australia

${ }^{3}$ Public Health and Wellbeing Research Group, Commonwealth Scientific and Industrial Research Organisation, Adelaide, Australia

Conflict of Interest Disclosure: The authors have not stated any conflicts of interest.

Address for correspondence: Jillian Claire Ryan, PhD, Precision Health Future Science Platform, Commonwealth Scientific and Industrial Research Organisation, 13 Kintore Ave, Adelaide, Australia 5000; E-mail: jillian.ryan@csiro.au

(C) 2021 The Authors. Published by Elsevier Inc. on behalf of Society for Nutrition Education and Behavior. This is an open access article under the CC BY-NC-ND license (http:// creativecommons.org/licenses/by-nc-nd/4.0/)

https://doi.org/10.1016/j.jneb.2021.08.001
}

vegetables, grains, proteins, and locally sourced foods has declined. ${ }^{1}$ These trends contradict health advice, which generally recommends a balanced diet consisting of an appropriate amount of energy; predominantly consisting of vegetables, fruits, whole grains as well as moderate amounts of lean meat and dairy or alternatives; and limited consumption of discretionary foods and beverages that are high in added saturated fat, salt, or added sugar. ${ }^{2}$ However, $<10 \%$ of Australian adults consume the recommended 5-6 servings of vegetables per day, ${ }^{3}$ a statistic mirrored by American adults. ${ }^{4}$ Meanwhile, discretionary foods are overconsumed, contributing a concerning one-third of total dietary 
energy intake, with little nutritional benefit. $^{3}$

The public health impacts of unhealthy diets are well-established. Overconsumption of calories and risk ingredients (such as salt and ultra-processed foods) and underconsumption of fruits and whole grains are drivers for diet-related chronic diseases, which are leading causes of death, illness, and disability. ${ }^{5}$ Furthermore, unhealthy dietary behaviors, particularly overconsumption of red meat and discretionary foods, also exacerbate global environmental problems such as the biodiversity crisis and climate change. ${ }^{6}$ In Australia, the agricultural industry is the largest contributor to environmental change through greenhouse gas emissions, freshwater use, and land degradation. ${ }^{6}$ Similarly, discretionary food consumption accounts for a significant proportion of diet-related greenhouse gas emissions, ${ }^{7}$ and freshwater use. $^{8}$

The growing body of evidence regarding the significant public health and environmental implications of unhealthy diets has led to the promotion of dietary patterns that are both healthy and environmentally sustainable. Notable indications include the EAT-Lancet Planetary Health Diet, which presents global dietary targets ${ }^{6}$ and calls for greater consideration of environmental sustainability within national dietary guidelines. ${ }^{9}$ However, changing dietary behaviors is difficult, and unhealthy patterns persist despite significant national health promotion and community education programs. Further investigation of the underlying drivers for eating patterns and how to effectively promote healthy dietary behaviors is needed.

Self-identity-based intervention tailoring may offer a potentially powerful but underused means to boost the appeal, relevance, and therefore effectiveness of dietary behavior interventions. Self-identity is a central component of our internal selves that is thought to consist of 2 main components: the individual identity, which Erikson described as a persistent sameness within oneself, and the social identity, consisting of persistent sharing of core characteristics with others. ${ }^{10}$ Because dietary behaviors require continual attention and effort (behavioral persistence) and have important social implications, including for group membership, diet and self-identity are deeply intertwined for many people. ${ }^{11-13}$ For example, it is common for individuals to identify with a dietary pattern label that depicts their overall dietary patterns (eg, vegetarian, vegan, or healthy diet), a process of self-definition. ${ }^{14}$ These selfdescribed dietary patterns have been shown to be strongly predictive of food choices; however, they are not set in stone, and people may stray from their self-defined dietary patterns. ${ }^{15}$

Rosenfeld and Burrow's ${ }^{15-17}$ operationalization of diet-related selfidentity is a key theoretical model within this topic. The model comprised 8 internal and social variables of diet-related self-identity, including measures of strictness (how flexible or inflexible one's diet is in different circumstances), motivation (whether diet choices are motivated by personal, prosocial, or moral factors) and social norms (the influence of social norms and group membership on dietary factors). ${ }^{15-17}$ Research based on this model has found that diets centered on the restriction of animal protein tend to be driven by prosocial motivations, such as a drive to minimize individual environmental impacts or empathy and concern toward nonhuman animals. ${ }^{16}$ Seeking improvements in health and subjective well-being is also a common motivating factor for choosing a vegetarian or vegan diet because of the health benefits of consuming low or no animal protein and high quantities of fruits, vegetables, whole grains, and legumes. ${ }^{18,19}$ The latter reflects a personal rather than prosocial motivation.

Predictive relationships between self-identity and diet have been wellstudied in relation to certain dietary patterns, including health-focused, weight loss, ${ }^{14}$ or plant-based. ${ }^{15}$ One study showed that among 343 college students in the US, self-identity and the extent to which an individual saw fruit and vegetable consumption as a core part of their identity was the strongest predictor of intention to eat fruit and vegetables over and above attitude, past behavior, perceived behavioral control, and subjective norms. ${ }^{20}$ In contrast, some dietary patterns, including intermittent fasting or ketogenic diets, have received relatively little research attention. The social and psychological drivers for these dietary patterns are poorly understood, as are the variety of diets that exist in different populations and their associations with health behavior. ${ }^{17,21}$ Self-identity has a temporal component that changes with shifts in societal, community, and individual values, which are sometimes evident in dietary trends, for example, fad diets and growing concern for the environmental impact of food choices. ${ }^{9}$ This study seeks to contribute to a better understanding of the role of diet-related self-identity in dietary behaviors. More specifically, the study aimed to assess the diversity of dietary patterns followed by a sample of Australian adults, determine how study participants with different dietary patterns differ in dietary behavior and diet self-identity factors, and investigate predictive relationships between dietary patterns and self-identity factors with diet quality.

\section{METHODS}

A cross-sectional study, in which a sample of Australian adults completed an online survey at a single time point, was conducted to address the research aims. Cross-sectional research is appropriate for exploration and hypothesis generation related to dynamic and emerging phenomena, including identity in dietary choices. ${ }^{22}$ The study received ethics approval from the University of South Australian and CSIRO Human Research Ethics committees before commencement, and all participants provided informed consent prior to participating. The study has been reported according to Strengthening the Reporting of Observational Studies in Epidemiology guidelines. ${ }^{23}$

\section{Participants and Procedure}

A questionnaire was developed that captured participants' dietary patterns, diet quality, and diet-related self-identity constructs. Participant characteristics (eg, age and sex [male, 
female, other, prefer not to disclose]) were also captured. The questionnaire was pilot-tested with 22 participants that included dietitians $(n=3)$ and members of the general population (Australian adults, $n=19$ ). Minor amendments to the survey were made on the basis of this feedback to reduce participant burden and improve clarity. Eligible participants included adults aged $\geq 18$ years who were in Australia at the time of the study, proficient in the English language, and who had access to an internet-enabled device on which they could complete the online survey. Participants were recruited through a paid advertising campaign distributed via the online social network Facebook, which has $>16$ million monthly active users in Australia (total population 25 million) and is the most popular online social network. ${ }^{24}$ Campaign parameters were set to show the advertisement in the main news feed of Facebook users with no restrictions on the basis of participant age, sex, or interests.

Potential participants who came across the advertisement in their Facebook newsfeed could click on a link that would take them to the survey hosted on the online survey software, SurveyGizmo (version 5, Alchemer LLC, 2020). On completion of the survey, participants were offered the opportunity to provide their email addresses to receive a summary of the research and/or enter a random prize draw to win 1 of 10 e-gift cards worth \$72 (or AUD \$100).

\section{Main Outcome Measure}

The main outcome was diet quality, which was assessed as the level of compliance with the Australian Dietary Guidelines. Food group intake was measured using the Commonwealth Scientific and Industrial Research Organisation Healthy Diet Score survey. ${ }^{25}$ The survey captured respondents' frequency and quantity of food intake across 6 food groups: fruit, vegetables, bread and cereals, meat and alternative sources of protein, dairy, and discretionary foods, which was then compared against daily targets provided in Australian Dietary Guidelines. ${ }^{26}$ Responses were scored and age-normalized to produce a compliance score for each food group and an overall diet quality score. Scores on each of these constructs range from 0 to 100 , with higher scores indicating greater compliance with the guidelines. The Healthy Diet Score survey has demonstrated strong test-retest reliability with $r=>0.80$ for all food groups except discretionary foods $(r=0.63)$ and moderate validity (intraclass correlation with 24-hour dietary recalls (range, 0.10-0.63) in Australian adults. $^{25}$

\section{Other Measures}

Dietary pattern. A single item was included to assess participants' selfdefined dietary patterns. Participants could choose from 1 of 7 predetermined diet labels: vegan, ovovegetarian, lactovegetarian, pescatarian, pescapollotarian, flexitarian/reducetarian, omnivore, none, or type in another dietary pattern. This approach of asking participants to identify their diet or dietary pattern has been used in previous research to capture the self-definition of dietary patterns. ${ }^{16,17,26,27}$

Diet self-identity constructs. Four diet self-identity constructs including prosocial motivation or the extent to which the respondent is driven to have a positive impact on the world around them, personal motivation or the extent to which one is driven by self-improvement, centrality, or the extent to which one's dietary pattern is central to their self-identity, and strictness or how strictly the respondent follows their dietary pattern, were captured using the relevant subscales from Rosenfeld and Burrow's Dietary Identity Questionnaire. ${ }^{17} \mathrm{Re}$ sponses were recorded on 7-point Likert scales. Mean scores on each subscale were calculated, with higher scores indicating stronger endorsement of each construct. The Diet Identity Questionnaire has demonstrated psychometric properties including internal consistency of $\alpha>0.80$ for all subscales $(n=290)$, and test-retest reliability of Pearson $r$ for each subscale ranging between $r=0.62$ and $0.80(\mathrm{n}=115){ }^{17}$

\section{Data Analysis}

Open-ended data capturing participants' dietary patterns were cleaned and coded using a content analysis approach. $^{28}$ The majority of dietary patterns were captured in the prespecified options (eg, omnivore, vegetarian). These open-ended text responses were thematically analyzed in duplicate by 2 authors and collapsed together when appropriate (eg keto or ketogenic diet). Disagreements were resolved through discussion with a third member of the authorship team. In total, 18 unique diet self-identities were recorded. Frequency counts of participants' dietary self-identities were calculated with those represented by $<5$ participants categorized into a general group (ie, other).

Next, the 18 diet self-identities were categorized into 3 groups to facilitate between-group inferential analyses. This process was completed by 2 qualified dietitians (K.A. and G.H.) from the authorship team and involved the iterative thematic analysis of each of the diet self-identities and duplicate classification of each of the diet self-identities, resulting in the following categories: (1) unrestricted diet (meat, dairy, etc), which included those that did not report to restrict foods from the core food groups (grains, dairy, meat, vegetables, or fruit); however, participants may (or may not) restrict energy intake or discretionary foods. The eating patterns may emphasize specific foods or nutrients such as olive oil or low-fat products or characteristics of foods such as processing type. Examples include intermittent fasting, Mediterranean dietary pattern, or the Australian Guide to Healthy Eating; (2) Dietary restriction (plantbased), which included dietary patterns focused on a plant-based pattern of eating and the restriction in consumption of meat/fish and other animal proteins. Examples include vegetarians or vegans who completely avoid meat and/or all animal products, flexitarians who only eat meat on a few days per week or special occasions, and people who avoid 1 type of meat product but not another (eg pescatarian); (3) Dietary restriction (other), which includes 
dietary patterns that restricted core foods other than animal-derived foods, such as foods from the core food groups (grains, dairy, meat, vegetables, fruit). In addition, they may (or may not) restrict energy intake or discretionary foods. Examples include ketogenic diets, low fermentable oligosaccharides, disaccharides, monosaccharides, and polyols diets; low carbohydrate diets; or the carnivore diet.

\section{Quantitative Data Analysis}

All analyses were conducted with SPSS (version 21; IBM, Armonk, NY). Results were considered statistically significant when $\alpha<0.05$. Quantitative data were analyzed descriptively, with mean (M) and SD calculated for continuous data and frequency counts calculated for categorical data. An ordinary least squares regression was conducted to identify significant predictors of diet quality. Data were first examined and confirmed to meet model assumptions, including linearity, independence of errors, homoscedasticity, and normality of residuals. ${ }^{29}$ Four outliers that had standardized residuals $>3$ were removed from the dataset before analysis. An ordinary least squares regression was undertaken with diet quality entered as dependent variable and sex, age, dietary pattern, diet strictness, centrality, prosocial, and personal diet motivations entered as independent variables. Dietary patterns were dummycoded such that plant-based dietary restrictions and other dietary restrictions were entered as dichotomous variables with an unrestricted dietary pattern as the reference category.

\section{RESULTS}

This study included a total of 2010 participants (Table 1). On average, participants were aged 52.1 years (SD, 16.0). Fifty-four percent of participants had completed a university degree, and the majority resided in the Australian states of New South Wales (29\%), Victoria (25\%), or Queensland (18\%).

\section{Variety of Dietary Patterns}

Unrestricted was the most common dietary pattern category, reported by just more than half of participants
(Figure 1). The next most common category of dietary patterns was a restriction of animal proteins, comprising approximately $37 \%$ of respondents. This group's specific dietary patterns included vegan (14\% of participants) and flexitarian-reducetarian (12\% of participants). Finally, dietary patterns based on restrictions of other foods comprised a total of $10 \%$ of participants and included dietary patterns such as ketogenic ( $2 \%$ of participants) or celiac/gluten-free $(<1 \%)$.

\section{Differences in Participant Characteristics Based on Dietary Patterns}

Dietary restriction (other) was the diet pattern category associated with the lowest overall diet quality scores $(\mathrm{M}$, 45.4; SD, 14.3) (Table 1). Plant-based dietary restriction was associated with the highest diet quality $(\mathrm{M}, 61.5$; SD, 13.7), whereas unrestricted diets fell in the middle (M, 56.4; SD, 14.1). Participants also differed in their consumption of foods from the different food groups (Figure 2). Plant-based dietary restriction was associated with the highest compliance scores for fruit and

Table 1. Demographic and Diet Self-Identity Characteristics of a Sample of Australian Adults Categorized by Dietary Pattern

Characteristics
Age, mean (SD)
Sex (\% female)
Psychosocial dimensions
of diet self-identity
Centrality
Prosocial
Personal
Strictness
Dietary compliance ${ }^{\mathrm{b}}$
Overall diet quality
Vegetables
Fruit
Bread and cereals
Meat and alternatives
Dairy
Discretionary foods

Diet Self-Identity Category

\begin{tabular}{|c|c|c|}
\hline $\begin{array}{l}\text { Category 1: } \\
\text { Unrestricted } \\
(n=1,045)\end{array}$ & $\begin{array}{l}\text { Category 2: } \\
\text { Plant-Based } \\
(n=723)\end{array}$ & $\begin{array}{l}\text { Category } 3 \text { : } \\
\text { Other } \\
(n=185)\end{array}$ \\
\hline $55.1(15.0)$ & $47.1(16.8)$ & $53.6(14.5)$ \\
\hline $55.3 \%$ & $69.8 \%$ & $62 \%$ \\
\hline $3.6(1.5)$ & $5.1(1.4)$ & $4.7(1.4)$ \\
\hline $3.3(1.4)$ & $5.5(1.2)$ & $3.8(1.5)$ \\
\hline $5.1(1.4)$ & $5.9(1.0)$ & $6.3(0.8)$ \\
\hline $2.9(1.1)$ & $4.7(1.9)$ & $4.2(1.7)$ \\
\hline $56.4(14.1)$ & $61.5(13.7)$ & $45.3(14.3)$ \\
\hline $70.8(28.1)$ & $83.2(23.3)$ & $51.9(39.4)$ \\
\hline 60.7 (38.2) & 76.9 (33.2) & $32.6(38.1)$ \\
\hline $62.8(24.6)$ & $66.1(23.1)$ & $21.8(30.8)$ \\
\hline 77.3 (23.8) & $67.1(28.1)$ & $67.1(28.1)$ \\
\hline $42.1(23.5)$ & $32.5(23.7)$ & $30.8(22.1)$ \\
\hline $24.8(30.7)$ & $42.7(34.0)$ & $47.5(37.6)$ \\
\hline
\end{tabular}

${ }^{\text {aPossible score range: } 1-7 ;{ }^{\text {b }} \text { Possible score range: } 0-100 .}$

Note: statistically significant differences in participant characteristics were identified between diet self-identity categories for all variables tested $(P \leq 0.001)$. Statistical significance was tested by 1 -way ANOVA (continuous variables) and chi-square test of independence (sex). 


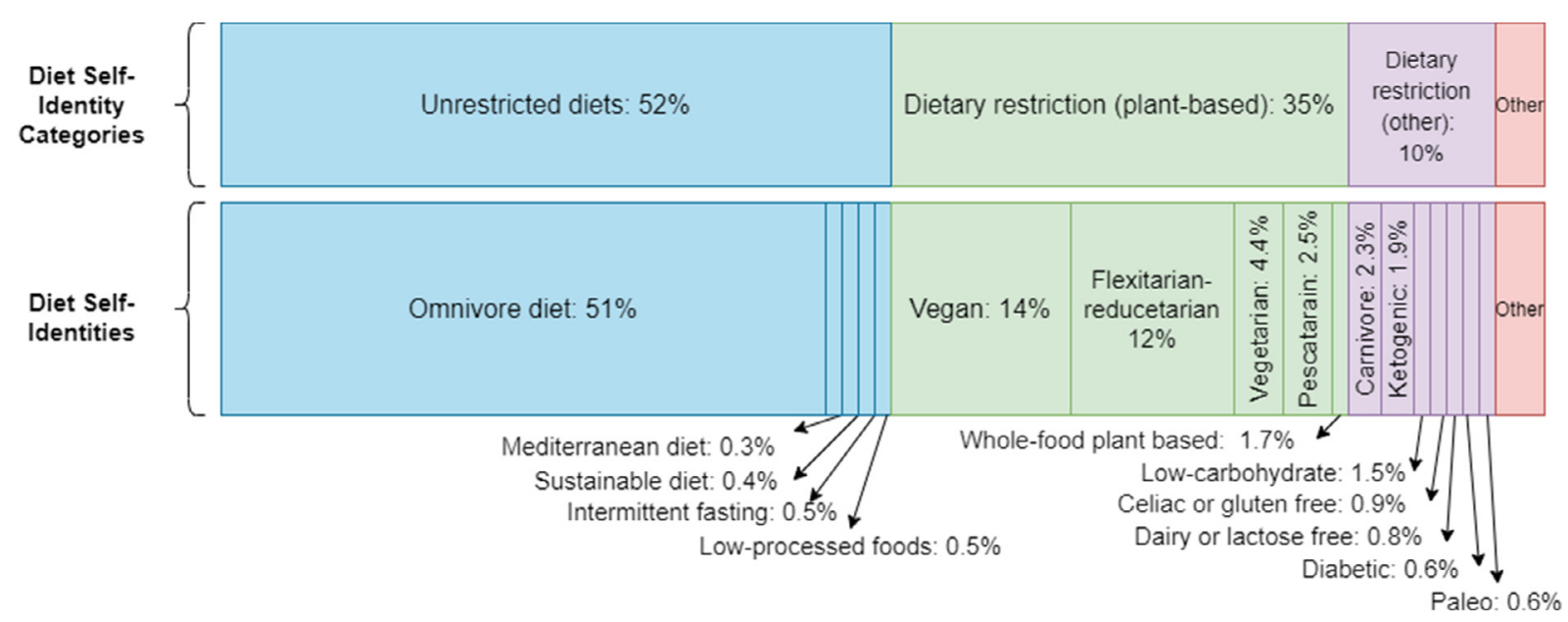

Figure 1. Distribution of Australian adults' self-reported diet-related self identities (July 21).

vegetable intake, whereas the other restriction group was associated with the lowest scores for fruits, vegetables, and bread/cereals. Unrestricted diets were associated with the lowest scores for discretionary food intake.

\section{Diet-Related Self-Identity and Dietary Pattern as Predictors of Diet Quality}

The ordinary least squares regression significantly predicted diet quality $(F$ $[8,1974]=54.952 ; \quad P<0.0001 ;$ adjusted $R^{2}=0.179$ ). Five of the 8 variables added statistically significantly to the prediction $(P<0.05)$. Age, personal motivations, and prosocial motivations were positively associated with diet quality, whereas the other restriction group was negatively associated with diet quality. Regression coefficients and standard errors can be found in Table 2 .

\section{DISCUSSION}

Diet-related health risk factors are a leading modifiable health risk factor. Because self-identity is an important determinant of health behaviors, it may offer a novel approach for tailoring dietary advice or interventions. Our findings suggest that Australians follow a diverse range of dietary patterns and are strongly linked to their self-identities and diet quality. Dietary patterns based on restriction of animal products or any other core food groups, which encompassed nearly half of all participants, reported lower consumption of discretionary foods compared with those with unrestricted diets. Plant-based dietary patterns, including vegetarian, and flexitarian were relatively common and associated with a greater intake of vegetables.

Similar to other countries around the world, Australians, in general, have poor diet quality, creating a need for new ways to shift intake toward a healthier pattern ${ }^{5}$; however, food choices are entrenched and difficult to change. Therefore, novel approaches are needed. ${ }^{30}$ In addition,

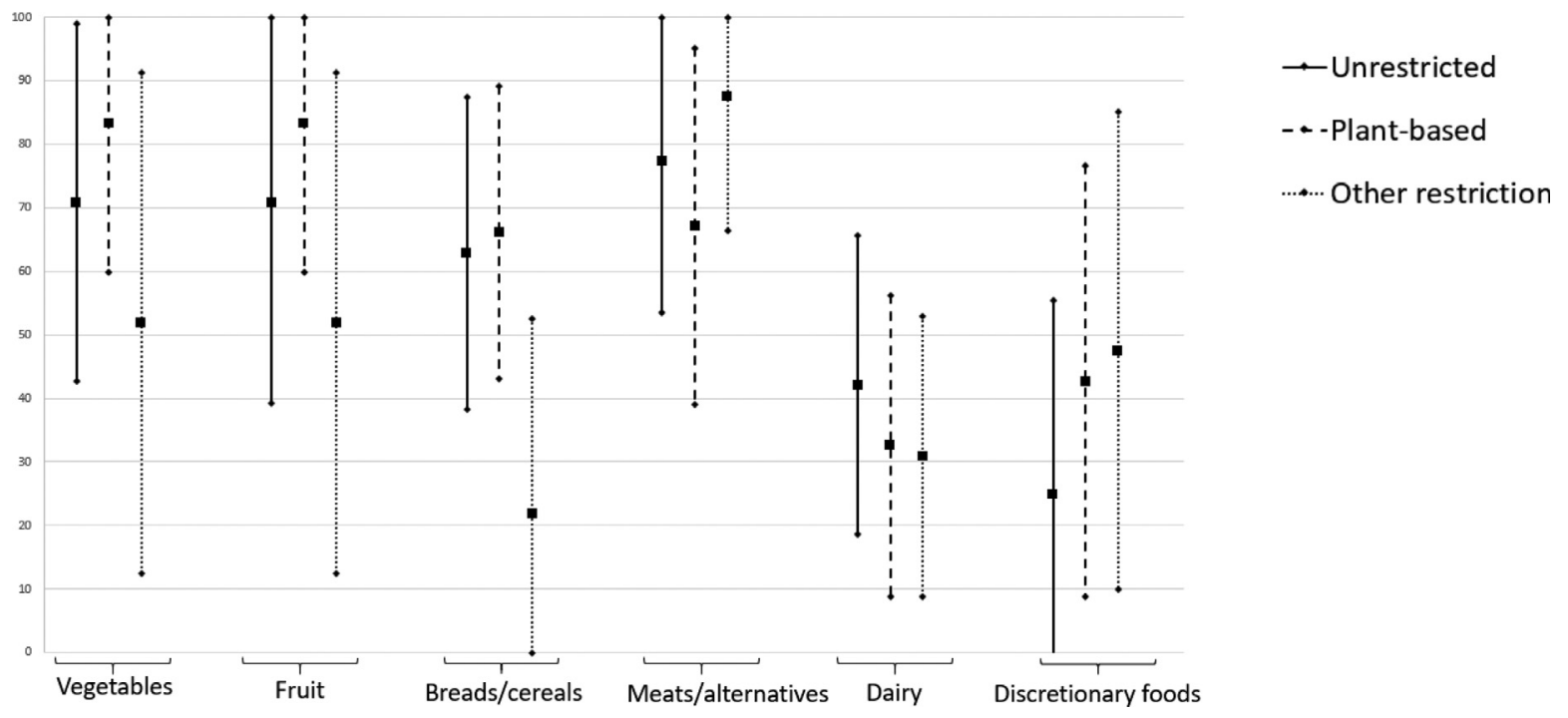

Figure 2. Between-groups differences in mean (central square) and standard deviations (whisker ends) dietary compliance scores among a sample of Australian adults. 
95\% Confidence Interval for $B$

\begin{tabular}{|c|c|c|c|c|c|c|c|}
\hline Diet Quality & $B$ & Lower Limit & Upper Limit & SE $B$ & $\boldsymbol{\beta}$ & $R^{r}$ & $\Delta \boldsymbol{R}^{r}$ \\
\hline Model & & & & & & 0.182 & $0.179^{* * *}$ \\
\hline Constant & 32.0558 & 28.472 & 36.644 & 2.093 & & & \\
\hline Age & 0.157 & 0.119 & 0.195 & 0.020 & $0.170^{* * *}$ & & \\
\hline Sex & -0.020 & -1.247 & 1.206 & 0.626 & -0.001 & & \\
\hline Diet pattern (restricts animal protein) & 3.057 & 1.374 & 4.739 & 0.858 & $0.099^{* \star *}$ & & \\
\hline Diet pattern (restricts other food type) & -13.944 & -16.170 & -11.717 & 1.135 & $-0.275^{\star \star \star}$ & & \\
\hline Centrality & 0.061 & -0.426 & 0.547 & 0.248 & 0.007 & & \\
\hline Strictness & -0.054 & -0.463 & 0.354 & 0.208 & -0.006 & & \\
\hline Prosocial motivation & 0.627 & 0.117 & 1.138 & 0.260 & $0.073^{*}$ & & \\
\hline Personal motivation & 2.538 & 2.002 & 3.073 & 0.273 & $0.226^{* *}$ & & \\
\hline
\end{tabular}

${ }^{\star} P<0.05 ;{ }^{* \star} P<0.01 ;{ }^{* \star \star} P<0.001$.

Note: Results from ordinary least squares regression.

the environmental impacts of food choices and the food supply are significant. ${ }^{6}$ Findings from the current study suggest a relatively higher proportion of people following vegan and vegetarian diets $(18.4 \%$ of participants) than previously reported in Australia. ${ }^{31}$ This may reflect a sampling bias because people who are more interested in the topic of food are more likely to volunteer to participate in a food-focused survey. However, the prevalence of vegetarianbased diets in Australia is not directly measured in national health surveys, so there is not a robust figure to make a comparison. The national Australian Health Survey asks about avoiding foods for cultural or ethical reasons, and in the latest version (2011-13), it is reported that about $2 \%$ and $4 \%$ of people avoid meat and pork, respectively, for these reasons. ${ }^{32}$ Another informal survey of $>$ 25,000 respondents suggested that around $4 \%$ of Australians were vegan or vegetarian, whereas $6 \%$ were semivegetarian. ${ }^{33}$ Capturing the prevalence of different dietary patterns using systematic sampling methods is important for future research.

Although restricting/avoiding food types other than animal proteins was the least-common diet self-identity (eg, ketogenic, paleo, and diabetic diets), it is still important to consider these diets given their potential health and environmental impacts. These diet selfidentities were associated with higher consumption of protein-based foods with less emphasis on high fiber foods such as fruits, vegetables, and whole grains. This may be problematic longer-term, given the benefits of high fiber foods in preventing chronic diseases. In addition, the emphasis of meat products high in saturated fat and exclusion of food groups such as dairy may make it difficult to meet nutrient requirements for a balanced and healthy diet. Furthermore, consuming meat in excess does not align with current recommendations for environmentally sustainable diets and may further exacerbate agricultural-related environmental pressures. ${ }^{6}$

Previous research has found that tailored health promotion initiatives are more effective than general health advice by providing only the information that is relevant for the target audience or individual's situation. ${ }^{34}$ Our findings highlight the need for messaging aligned with aspects of the target audience's selfidentity, dietary patterns, personal and prosocial motivations. This is consistent with previous research finding that messages aligned to participants' perceptions of different social norms ${ }^{14}$ and goals ${ }^{35}$ are more effective than one-size-fits-all approaches.

A major strength of the study is the large sample $(n=2,010)$, which can help to control for the risk of a type II reporting error (false negative finding) and offer findings that are more generalizable to the broader Australian public. Further bolstering the generalizability of these results is the relatively balanced participant sociodemographic information such that both males and females across varying education levels and different states of Australia were represented. This is important because gender and education remain key factors in explaining diet choice. ${ }^{36}$ Furthermore, this study drew on validated scales as measurement tools, which may not always be the case in this research area, leading to a deeper and less biased understanding of diet-self-identity.

However, there are potential limitations of the research that are important to acknowledge. The recruitment approach of using paid Facebook advertising campaigns may have inadvertently introduced a source of selection bias. Although Facebook has a wide and active membership in Australia, a concealed algorithm is used to determine which profiles see each advertisement; therefore, distribution may not be totally random. Although the advertising copy and imagery were designed to be as general as possible by focusing on food rather than any of the downstream implications of food consumption, it is still possible that outcomes overrepresent the broader Australian community as those who completed the online survey may be more interested in food than those who did not. Social desirability bias (ie, participants providing socially favorable survey responses ${ }^{37}$ ) can also affect results. For example, it is possible that participants over-stated rather than under-stated their diet 
self-identity-related motivations, strictness, and centrality, as well as their food intake. The limitations of selfreport data are recognized in the area of nutrition research, such as energy intake, $^{38}$ diet quality, ${ }^{39}$ and links between nutrition and health, ${ }^{40}$ and diet and sleep quality. ${ }^{41}$ Nevertheless, the use of self-report measures provides an opportunity to gather valuable and rich information. ${ }^{38}$ Another factor that may be addressed in future research is how diet-related self-identity was operationalized. This simple and purpose-developed tool did not consider that people may follow more than 1 variation in the extent of respondents' commitment/compliance to their diet selfidentity.

\section{IMPLICATIONS FOR RESEARCH AND PRACTICE}

Understanding the values underpinning diet-related self-identity and variation in how diets are expressed can provide vital insights to inform tailored dietary advice and interventions with public health or environmental focus. Given that self-identity provides an enduring perception of one's self that is also highly predictive of behavior over time, ${ }^{12}$ nutrition initiatives may also benefit from identity-based audience segmentation and the design of pragmatic interventions that work with, rather than against, an individual's current eating habits. Helping people identify and refine their self-identities to be more aligned with their food and health goals may represent a new opportunity for dietary behavior interventions seeking longer-term or sustained effects. Further research is needed that evaluates the population prevalence of different dietary patterns among representative research samples. Potential implications of this finding include that messages targeting social benefits of dietary patterns may be more relevant to people who follow a plant-based diet, whereas health- or beauty-focused messages may resonate strongly with people who follow a dietary pattern focused on the restriction of another core food group. Further research to explore this possibility is needed.

\section{ACKNOWLEDGMENTS}

The authors would like to thank the participants in this study, including the pilot study participants who provided feedback on the survey instrument.

\section{REFERENCES}

1. Zobel EH, Hansen TW, Rossing P, von Scholten BJ. Global changes in food supply and the obesity epidemic. Curr Obes Rep. 2016;5:449-455.

2. World Health Organization. Healthy Diet. World Health Organization. Regional Office for the Eastern Mediterranean; 2020.

3. Australian Institute of Health and Welfare. Nutrition Across the Life Stages. Australian Government; 2018.

4. Lee-Kwan SH, Moore LV, Blanck HM, Harris DM, Galuska D. Disparities in state-specific adult fruit and vegetable consumption-United States, 2015. MMWR Morb Mortal Wkly Rep. 2017; 66:1241.

5. Afshin A. Health effects of dietary risks in 195 countries, 1990-2017: a systematic analysis for the Global Burden of Disease Study 2017. Lancet. 2019; 393:1958-1972.

6. Willett W, Rockström J, Loken B, et al. Food in the Anthropocene: the EAT -Lancet Commission on healthy diets from sustainable food systems. Lancet. 2019;393:447-492.

7. Hendrie G, Baird D, Ridoutt B, Hadjikakou M, Noakes M. Overconsumption of energy and excessive discretionary food intake inflates dietary greenhouse gas emissions in Australia. Nutrients. 2016;8.

8. Ridoutt BG, Baird D, Anastasiou K, Hendrie GA. Diet quality and water scarcity: evidence from a large Australian population health survey. Nutrients. 2019;11.

9. Reynolds CJ, Buckley JD, Weinstein P, Boland J. Are the dietary guidelines for meat, fat, fruit and vegetable consumption appropriate for environmental sustainability? A review of the literature. Nutrients. 2014;6:2251-2265.

10. Erikson EH. The problem of ego identity. J Am Psychoanal Assoc. 1956;4:56121.

11. Terry DJ, Hogg MA, White KM. The theory of planned behaviour: self-identity, social identity and group norms. $\mathrm{Br}$ J Soc Psychol. 1999;38:225-244.
12. Cruwys T, Norwood R, Chachay VS, Ntontis E, Sheffield J. "An important part of who I am": the predictors of dietary adherence among weight-loss, vegetarian, vegan, paleo, and gluten-free dietary groups. Nutrients. 2020;12:970.

13. Chuck C, Fernandes SA, Hyers LL. Awakening to the politics of food: politicized diet as social identity. Appetite. 2016;107:425-436.

14. Yun D, Silk KJ. Social norms, self-identity, and attention to social comparison information in the context of exercise and healthy diet behavior. Health Commun. 2011;26:275-285.

15. Rosenfeld DL, Burrow AL. Vegetarian on purpose: understanding the motivations of plant-based dieters. Appetite. 2017;116:456-463.

16. Rosenfeld DL, Burrow AL. The unified model of vegetarian identity: A conceptual framework for understanding plant-based food choices. Appetite. 2017;112:78-95.

17. Rosenfeld DL, Burrow AL. Development and validation of the Dietarian Identity Questionnaire: assessing selfperceptions of animal-product consumption. Appetite. 2018;127:182-194.

18. Fox N, Ward KJ. You are what you eat? Vegetarianism, health and identity. Soc Sci Med. 2008;66:2585-2595.

19. Marsh K, Zeuschner C, Saunders A. Health implications of a vegetarian diet: a review. Am J Lifestyle Med. 2012; 6:250-267.

20. Jung SE, Bice C. The role of self-identity in predicting college students' intention to consume fruits and vegetables. $J$ Nutr Educ Behav. 2019;51:173-181.

21. Norwood R, Cruwys T, Chachay VS, Sheffield J. The psychological characteristics of people consuming vegetarian, vegan, paleo, gluten free and weight loss dietary patterns. Obes Sci Pract. 2019;5:148-158.

22. Mann C. Observational research methods. Research design II: cohort, cross sectional, and case-control studies. Emerg Med J. 2003;20:54-60.

23. Von Elm E, Altman DG, Egger M, Pocock SJ, Gøtzsche PC, Vandenbroucke JP. The Strengthening the Reporting of Observational Studies in Epidemiology (STROBE) statement: guidelines for reporting observational studies. Ann Intern Med. 2007;147:573-577.

24. Roy Morgan. Facebook on top but Instagram and Pinterest growing fastest. http://www.roymorgan.com/findings/ 7979-social-media-trends-march-2019- 


\section{Accessed October 3, 2019.}

25. Hendrie GA, Baird D, Golley RK, Noakes M. The CSIRO healthy diet score: an online survey to estimate compliance with the Australian dietary guidelines. Nutrients. 2017;9:47.

26. Pfeiler TM, Egloff B. Do vegetarians feel bad? Examining the association between eating vegetarian and subjective well-being in two representative samples. Food Qual Preference. 2020;86:104018.

27. Hargreaves SM, Nakano EY, Zandonadi RP. Brazilian vegetarian populationinfluence of type of diet, motivation and sociodemographic variables on quality of life measured by specific tool (VEGQOL). Nutrients. 2020;12:1406.

28. Neuendorf KA. The Content Analysis Guidebook. SAGE Publications; 2017.

29. Casson RJ, Farmer LD. Understanding and checking the assumptions of linear regression: a primer for medical researchers. Clin Exp Ophthalmol. 2014; 42:590-596.

30. de Ridder D, Kroese F, Evers C, Adriaanse M, Gillebaart M. Healthy diet: health impact, prevalence, correlates, and interventions. Psychol Health. 2017;32:907-941.
31. Roy Morgan. The Slow but Steady Rise of Vegetarianism in Australia. Roy Morgan Research Press Release; 2016.

32. Australian Bureau of Statistics. Australian health survey: nutrition first results - foods and nutrients. https://www.abs. gov.au/statistics/health/health-conditions-and-risks/australian-health-survey-nutrition-first-results-foods-andnutrients/latest-release. Accessed September 6, 2021.

33. Sutton M. Vegans a 1 per cent minority in a country of meat eaters, survey finds. https://www.abc.net.au/news/2019-1026/vegans-comprise-just-1-per-cent-ofthe-population-survey-finds/11635306. Accessed September 6, 2021.

34. De Bourdeaudhuij I, Brug J. Tailoring dietary feedback to reduce fat intake: an intervention at the family level. Health Educ Res. 2000;15:449462.

35. Dominick JK, Cole S. Goals as identities: boosting perceptions of healthyeater identity for easier goal pursuit. Motiv Emot. 2020;44:410-426.

36. Mosier SL, Rimal AP. Where's the meat? An evaluation of diet and partisanship identification. $\mathrm{Br}$ Food $\mathrm{J}$. 2019;122:896-909.
37. King MF, Bruner GC. Social desirability bias: A neglected aspect of validity testing. Psychol Mark. 2000;17:79-103.

38. Subar AF, Freedman LS, Tooze JA, et al. Addressing current criticism regarding the value of self-report dietary data. J Nutr. 2015;145:2639-2645.

39. Warren-Findlow J, Reeve CL, Racine EF. Psychometric validation of a brief self-report measure of diet quality: the DASH-Q. J Nutr Educ Behav. 2017;49: 92-99.e1.

40. Kirkpatrick SI, Vanderlee L, Raffoul A, et al. Self-report dietary assessment tools used in Canadian research: a scoping review. Adv Nutr. 2017;8:276-289.

41. Godos J, Grosso G, Castellano S, Galvano F, Caraci F, Ferri R. Association between diet and sleep quality: a systematic review. Sleep Med Rev. 2021:101430.

\section{ORCIDs}

Jillian Claire Ryan: http://orcid.org/ 0000-0003-0655-3047

Kim Anastasiou: http://orcid.org/

0000-0002-3338-5117

Carla Litchfield: http://orcid.org/

0000-0002-1167-1374 